\title{
Body Weight Perception Versus Body Weight Status: How Good is The Agreement?
}

Nurjasmine Aida binti Jamani ${ }^{1}$, Abdul Hadi bin Said ${ }^{1}$, Karimah Hanim binti Abd.Aziz ${ }^{2}$, Mohd Aizuddin bin Abd Rahman ${ }^{1}$

${ }^{1}$ Department of Family Medicine, Kulliyyah of Medicine, International Islamic University Malaysia

${ }^{2}$ Department of Community Medicine, Kulliyyah of Medicine, International Islamic University Malaysia

Presenter: Mohd Aizuddin bin Abd Rahman

Introduction: Body weight perception is an important underlying psychological factor associated with body weight. Overemphasis on thinness among adolescents can lead to unhealthy dieting practices and eating disorders, whereas underestimation of body weight may increase risk of the development of overweight and obesity. This study measured the agreement between body weight perception and body weight status (BMI) among late adolescents in Kuantan. Materials and Methods: A cross-sectional study was conducted from April to August 2018 among 418 adolescents of six higher learning institutions in Kuantan; aged 17-19 years old. Weight and height were measured and BMI was calculated. Self-administered, validated questionnaires; Figure Rating Scale (FRS) and International Physical Activity Questionnaire (IPAQ) were used. The strength of agreement between BMI and body weight perception was examined using Kappa statistics. Results: Overall, $37.6 \%$ of total participants misjudged their own body weight. Of particular note, $51.9 \%$ of real underweight participants misperceived body weight as normal weight and overweight while $47.2 \%$ of obese participants incorrectly perceived their body weight as overweight. The agreement between body weight perception and body mass index was poor $(k=-0.09, p<0.01)$. The discrepancy more marked in male $(k=-0.11, p<0.01)$. Conclusions: Body weight perception was not in agreement with actual weight in late adolescents. Accurate body weight perception is an important factor in late adolescent's weight management behaviour. More effort should be done in order to correct misperception among this age group for their health benefits. 